\title{
Capsule Commentary on Robinson et al.: A Randomized Trial on the Efficacy of Mastery Learning on Primary Care Providers' Melanoma Opportunistic Screening Skills and Practice
}

\author{
Ambarish M. Athavale, MBBS, MD \\ Division of Nephrology, Core Faculty, Internal Medicine Residency Program, John H. Stroger Jr. Hospital of Cook County, Chicago, IL, USA.
}

J Gen Intern Med 33(6):946

DOI: $10.1007 / \mathrm{s} 11606-018-4360-7$

(c) Society of General Internal Medicine 2018

$\mathrm{T}$ his randomized educational trial by Robinson et al. ${ }^{1}$ assessed the efficacy of Mastery learning course to improve Primary Care Physicians (PCPs) accuracy in diagnosing skin lesions with emphasis on melanoma detection. The Mastery learning course included "visual and dermoscopic assessment, diagnosis and management, and deliberate practice with feedback to reach a minimum passing standard". ${ }^{1}$ PCPs in the intervention arm detected more melanomas on standardized posttest and referred more melanomas and fewer benign lesions than in control group.

Prior studies have shown improvement in PCP skills with education focused on unaided visual assessment. ${ }^{2}$ This study demonstrates that PCPs can be effectively trained in dermoscopy $(\times 10$ magnification $)$ in addition to unaided visual inspection. Trained PCPs had greater diagnostic accuracy for referred lesions; however lesions not referred were not assessed for diagnostic accuracy and is a source of bias in this study. Assessment of knowledge/skill retention was not an objective of this study and it is not known how frequently PCPs need to be trained to maintain competency in screening. Further research in these areas is needed prior to routine adoption of this tool in clinical training/practice.

Incidence of melanoma has been increasing and the annual visit to the PCP is a good opportunity to screen for skin cancer. ${ }^{3}$ PCP diagnosed melanomas tend to be smaller, early stage and have better prognosis. ${ }^{3}$ However, prior studies have shown that PCPs feel that their training is inadequate and are not confident of their skills in diagnosing skin lesions. ${ }^{4}$ From the providers' perspective, educational interventions like the Mastery learning course fulfill a perceived need by providing an online self-paced learning tool. Such interventions are also likely to be cost-effective by increasing referral of early lesions and decreasing referral of benign lesions.

Corresponding Author: Ambarish M. Athavale, MBBS, MD; Division of Nephrology, Core Faculty, Internal Medicine Residency Program, John H. Stroger Jr. Hospital of Cook County, Chicago, IL, USA (e-mail: aathavale@cookcountyhhs.org).

\section{Compliance with Ethical Standards:}

Conflict of Interest: The author declares that there is no conflict of interest.

\section{REFERENCES}

1. Robinson JK, Jain N, Marghoob AA, McGaghie W, MacLean M, Gerami P, Hultgren B, Turrisi R, Mallett K, Martin GJ. A Randomized Trial on the Efficacy of Mastery Learning on Primary Care Providers' Melanoma Opportunistic Screening Skills and Practice. J Gen Intern Med. DOI: https://doi.org/10.1007/s11606-018-4311-3

2. Swetter SM, Chang J, Shaub AR, Weinstock MA, Lewis ET, Asch SM. Primary Care-Based Skin Cancer Screening in a Veterans Affairs Health Care System. JAMA Dermatol. 2017;153(8):797-801.

3. Mariah M Johnson, Sancy A Leachman, Lisa G Aspinwall, Lee D Cranmer, Clara Curiel-Lewandrowski et al. Skin cancer screening: recommendations for data-driven screening guidelines and a review of the US Preventive Services Task Force controversy. Melanoma Manag. 2017; 4(1): 13-37.

4. Friedman KP, Whitaker-Worth DL, Grin C, Grant-Kels JM. Melanoma screening behavior among primary care physicians. Cutis. 2004;74(5):305-11. 